02

\title{
Сдвиги частоты магнитного резонанса атомов Na при столкновении в смеси атомов калия и натрия
}

\author{
(C) B.A. Картошкин \\ Физико-технический институт им. А.Ф. Иофрфе РАН, \\ 194021 Санкт-Петербург, Россия \\ e-mail: victor.kart@mail.ioffe.ru
}

Поступило в Редакцию 24 марта 2021 г.

В окончательной редакции 25 апреля 2021 г.

Принято к публикации 26 апреля 2021 г.

Рассмотрены столкновения между атомами натрия и калия в условиях оптической ориентации атомов калия. В результате столкновений происходит спиновый обмен между сталкивающимися атомами, что приводит к сдвигу частоты магнитного резонанса атомов. Проведенные расчеты показали, что сдвиг частоты магнитного резонанса атомов $\mathrm{Na}$ в верхнем сверхтонком состоянии $(\mathbf{F}=2)$ при росте температуры в камере поглощения от 300 до $500 \mathrm{~K}$ меняет знак с отрицательного на положительный и проходит через нуль в окрестностях температуры $T \sim 450 \mathrm{~K}$, в то время как сдвиг частоты нижнего сверхтонкого состояния $(\mathbf{F}=1)$ остается отрицательным во всем диапазоне исследуемых температур. Подобного рода явление обнаружено впервые для случая, когда основной вклад в величину сдвига частоты магнитного резонанса вносит спиновый обмен.

Ключевые слова: спиновый обмен, поперечные сечения, сдвиги частоты магнитного резонанса.

DOI: 10.21883/JTF.2021.09.51209.78-21

\section{Введение}

В настоящее время большую роль в физических исследованиях играют устройства, в основе работы которых лежит принцип оптической ориентации атомов. В частности, при проведении разного рода магнитных измерений используются квантовые магнитометры с оптической накачкой $[1,2]$, оптическая ориентация атомов используется при создании квантовых гироскопов [3], а также магнитоэнцефалографов [4]. В качестве рабочих сред такого рода устройств выступают, в частности, атомы щелочных металлов в основном состоянии [5], причем как атомы одного сорта, так и смеси разных щелочных атомов [6,7]. Присутствующие в камере поглощения щелочные атомы сталкиваются друг с другом, при этом столкновения щелочных атомов сопровождаются хорошо известным процессом спинового обмена, т.е. процессом обмена электронной поляризацией между сталкивающимися атомами. Процесс спинового обмена существенным образом влияет как на ширину линии магнитного резонанса, так и на величины сдвигов частоты магнитного резонанса щелочных атомов [8], участвующих в столкновении.

Если один из сталкивающихся атомов был предварительно поляризован, то его поляризация может быть передана партнеру столкновения. Это, в частности, позволяет осуществить поляризацию партнера столкновения „непрямым“ образом без использования поляризованного резонансного оптического излучения. Подобного рода „непрямая“ поляризация используется, в частности, в ситуациях, когда „прямую“ оптическую ориентацию невозможно реализовать по тем или иным причинам, или когда необходимо „развязать“ каналы накачки и регистрации магнитного резонанса [9]. Известно, что процесс спинового обмена сопровождается как отмеченным выше процессом переноса поляризации, так и сдвигом частоты магнитного резонанса атомов. В случае, когда процесс спинового обмена происходит в смеси щелочных атомов, на величину сдвигов частоты магнитного резонанса влияют как столкновения одинаковых атомов между собой, так и столкновения с атомами другого сорта. В [10] были рассмотрены спин-обменные сдвиги частоты магнитного резонанса в смеси щелочных атомов в атмосфере буферного газа. В настоящей работе с опорой на данные работы [10] будут рассчитаны сдвиги частоты магнитного резонанса атомов $\mathrm{Na}$ в смеси атомов $\mathrm{K}$ и $\mathrm{Na}$ и построены их температурные зависимости, обусловленные процессом спинового обмена.

\section{1. Спин-обменные сдвиги частоты магнитного резонанса для смеси атомов щелочных металлов}

Атомы щелочных металлов, находящиеся в основном состоянии, обладают электронным спином $\mathbf{S}=1 / 2$. У рассматриваемых в настоящей работе атомов ${ }^{23} \mathrm{Na}$ имеется ядерный спин $\mathbf{I}_{\mathbf{N a}}=3 / 2$, у атомов ${ }^{39} \mathrm{~K}-\mathbf{I}_{\mathbf{K}}=3 / 2$. Как уже отмечалось выше, сдвиги частоты магнитного резонанса в смеси щелочных атомов были исследованы в [10]. В рассматриваемой модели в системе двух щелочных атомов $A$ и $B$ осуществляется оптическая ориентация атомов $B$ и определяются сдвиги 
частоты магнитного резонанса атомов $A$ для двух сверхтонких состояний. В процессе спин-обменных столкновений между атомами $A$ и $B$ ориентация передается атомам $A$. Эволюция матрицы плотности атомов $A$ в таком случае может быть описана следующим уравнением:

$$
\begin{aligned}
\frac{d}{d t}^{(A)} \rho= & {\left[\left(\frac{\partial}{\partial t}\right)_{A B}+\left(\frac{\partial}{\partial t}\right)_{A A}+\left(\frac{\partial}{\partial t}\right)_{A C}\right.} \\
& \left.+\left(\frac{\partial}{\partial t}\right)_{D}+\left(\frac{\partial}{\partial t}\right)_{H}\right]^{(A)} \rho .
\end{aligned}
$$

В соответствии с принятыми в [10] обозначениями имеем: первое слагаемое описывает столкновения разных атомов $(A$ и $B)$, второе - столкновения одинаковых атомов $(A$ и $A)$, третье - столкновения атомов $A$ с атомами буферного газа $C$, четвертое - диффузию к стенкам камеры поглощения, последнее - взаимодействие атомов с постоянным магнитным полем.

Можно рассмотреть влияние различных факторов, входящих в (1), и оценить их вклад в сдвиги частоты магнитного резонанса.

Учет влияния в кинетических уравнениях вклада, связанного с деполяризацией щелочных атомов на стенке камеры поглощения и в объеме, при столкновении с атомами буферного газа необходим, когда скорости спинового обмена $\bar{\gamma}_{A B}$ и $\bar{\gamma}_{A A}$ сравнимы со скоростью релаксации, обусловленной диффузией $\gamma_{D}$ и столкновениями с атомами буферных газов $\gamma_{A C}[10]$. Действительно, скорость диффузии определяется как

$$
\gamma_{D}=\frac{D}{\lambda_{D}^{2}}
$$

где $D$ - коэффициент диффузии щелочных атомов в буферном газе, $\lambda_{D}$ - диффузионная длина, определяемая из линейных размеров камеры поглощения (в стандартных для наших экспериментов по оптической ориентации атомов камерах поглощения диаметром 4 и длиной $6 \mathrm{~cm}$ получается $1 / \lambda_{D}^{2}=1.71 \mathrm{~cm}^{-2}$ при давлении буферного газа 100 Torr). При этом для случая использования в качестве буферного газа гелия или неона имеем следующие коэффициенты диффузии для атомов Na: коэффициент диффузии $D_{0}=(1.2 \pm 0.2) \mathrm{cm}^{2} \mathrm{~s}^{-1}$ для случая, когда буферным газом является Не, и $D_{0}=(0.67 \pm 0.05) \mathrm{cm}^{2} \mathrm{~s}^{-1}$ для случая, когда буферным газом является $\mathrm{Ne}$. Величины коэффициентов диффузии приведены для $180^{\circ} \mathrm{C}$ [11]. Коэффициент диффузии $D$ связан с $D_{0}$ следующим соотношением: $D_{0}=P / P_{0} D$, где $P$ - давление газа в камере поглощения, $P_{0}=760$ Torr. Таким образом, в соответствии с формулой (2) имеем $\gamma_{D}=9.12 \mathrm{~s}^{-1}$.

Вместе с тем, наличие буферного газа в камере поглощения приводит также к разрушению поляризации щелочного атома при столкновении с атомами буферного газа. Для атомов $\mathrm{Na}$ величина сечения деполяризации при столкновении с атомами $\mathrm{Ne}$ составляет в соответствии с данными $[11] \sigma_{A C}=(3.3 \pm 1.5) \cdot 10^{-24} \mathrm{~cm}^{2}$.
Отсюда можно определить скорость релаксации поляризованных атомов азота при столкновении с атомами буферного газа $-\gamma_{A C}=N_{C}\left\langle v_{A C}\right\rangle \sigma_{A C}$ (здесь $N_{C}-$ концентрация атомов буферного газа в камере поглощения, $\left\langle v_{A C}\right\rangle-$ средняя относительная тепловая скорость атомов, $\left\langle\sigma_{A C}\right\rangle-$ сечение деполяризации при столкновении атомов $\mathrm{Na}$ с атомами $\mathrm{Ne}$ ). В рассматриваемых условия эта величина равна $\gamma_{A C} \approx 1.07 \mathrm{~s}^{-1}$. Таким образом, при давлении буферного газа 100 Torr и температуре в камере поглощения $180^{\mid \text {circ }} \mathrm{C}$ мы имеем величину $\gamma_{D} \approx 8.7 \mathrm{~s}^{-1}$. Из данных работ $[12,13]$ следует, что скорость спинового обмена при столкновении атомов $\mathrm{Na}-\mathrm{Na}[12]$ и $\mathrm{Na}-\mathrm{K}$ [13] существенно выше, чем величины $\gamma_{D}$ и $\gamma_{A C}$, и вкладом от этих процессов в (1) можно пренебречь (третье и четвертое слагаемые в выражении (1)). Действительно, как следует из данных работ [12], сечение спинового обмена пары $\mathrm{Na}-\mathrm{Na}$ при $450 \mathrm{~K}$ равно $\sigma_{A A}=1.83 \cdot 10^{-14} \mathrm{~cm}^{2}$ (для потенциалов из работ $[14,15])$ и $\sigma_{A A}=1.58 \cdot 10^{-14} \mathrm{~cm}^{2}$ (для потенциалов из работы [15]) и соответственно для пары $\mathrm{Na}-\mathrm{K}-\sigma_{A B}=2.71 \cdot 10^{-14} \mathrm{~cm}^{2}$ из работы [13] (для потенциалов из работы [16]). Таким образом, при концентрации атомов $\mathrm{Na}$ в камере поглощения $N_{\mathrm{Na}}=9.1 \cdot 10^{11} \mathrm{~cm}^{-3}$ и $N_{\mathrm{K}}=1.3 \cdot 10^{13} \mathrm{~cm}^{-3}$ мы получаем следующие значения величин $\bar{\gamma}_{A B}=2.8 \cdot 10^{4} \mathrm{~s}^{-1}$ и $N_{\mathrm{K}}=1.67 \cdot 10^{3} \mathrm{~s}^{-1}$ (сечения с использованием потенциалов из [14-16]) и $\bar{\gamma}=1.23 \cdot 10^{3} \mathrm{~s}^{-1}$ (сечения с использованием потенциалов из [15]). Из приведенных величин видно, что основную роль в уравнении (1) играет процесс спинового обмена между атомами.

В случае использования в экспериментах камер поглощения с антирелаксационным покрытием деполяризации щелочных атомов на стенках камеры поглощения не происходит и, кроме того, буферный газ при изготовлении не используется, что исключает деполяризацию щелочных атомов на атомах (или молекулах) буферного газа. Таким образом, в этом случае третьим и четвертым слагаемыми в (1) также можно пренебречь.

При учете пятого члена в формуле (1) надо иметь ввиду, что, как правило, эксперименты по оптической ориентации проводятся в слабых магнитных полях, таким образом, нелинейностью расщепления зеемановских подуровней щелочного атома в магнитном поле можно пренебречь. Вместе с тем, выполняется условие, когда частота сверхтонкого расщепления $F=S \pm \mathbf{I}$ щелочного атома $\omega(\mathrm{F})$ много больше скорости спинового обмена [10].

Из вышесказанного следует, что при расчете величин сдвигов частоты магнитного резонанса можно воспользоваться выражениями, полученными без учета диффузии и деполяризации на буферном газе а также влияния постоянного магнитного поля.

В таком случае в соответствии с [10] учет только спин-обменных столкновений приводит к следующим выражениям для величин сдвигов частоты магнитного 
резонанса двух сверхтонких состояний щелочного атома:

$$
\Delta \omega( \pm)=\delta^{(1)} \omega( \pm)+\delta^{(2)} \omega .
$$

Здесь первое слагаемое обусловлено возникновением у атома $A$ добавки к поперечной компоненте ориентации в столкновениях с продольно ориентированными атомами $B$, а второй член обусловлен переносом поперечной ориентации с одного подуровня $\mathbf{F}$ атома $A$ на другой в результате столкновений. Представленные в (3) сдвиги в соответствии с $[10]$ имеют следующий вид:

$$
\begin{gathered}
\delta^{(1)} \omega(+)=-\frac{P_{z}(B)}{2\left(2 I_{A}+1\right)}\left[\overline{\bar{\gamma}}_{A B}-\overline{\bar{\gamma}}_{A A} B-\left(\frac{2 I_{A}-1}{2 I_{A}+1}\right)^{1 / 2}\right], \\
\delta^{(1)} \omega(-)=-\frac{P_{z}(B)}{2\left(2 I_{A}+1\right)}\left[\overline{\bar{\gamma}}_{A B}+\overline{\bar{\gamma}}_{A A} B_{+}\left(\frac{2 I_{A}+3}{2 I_{A}+1}\right)^{1 / 2}\right], \\
\delta^{(2)} \omega(+)=-\frac{C}{\bar{\omega}_{0}}\left\{\left(2 \bar{\gamma}_{A A}+3 \bar{\gamma}_{A B}\right)^{2}-\left[\overline{\bar{\gamma}}_{A A} P_{z}(B)\right]^{2}\right\} .
\end{gathered}
$$

В настоящей работе индекс $A$ относится к атомам ${ }^{23} \mathrm{Na}$, а индекс $B-$ к атомам ${ }^{39} \mathrm{~K}$. Далее $\delta^{(1)} \omega(+)-$ сдвиг частоты магнитного резонанса для сверхтонкого состояния $F=S+\mathbf{I}$ (где $S$ - электронный спин и в случае щелочных атомов он равен $1 / 2$, а I - ядерный спин щелочного атома (в нашем случае $\mathbf{I}=3 / 2)), \delta^{(1)} \omega(-)-$ сдвиг частоты магнитного резонанса для сверхтонкого состояния $F=S-\mathbf{I}, P_{z}(B)-$ поляризация частицы $B$ (как уже отмечалось выше, спин-обменные сдвиги рассчитываются, когда оптической ориентации подвергнуты атомы $B$, в нашем случае это атомы $\left.{ }^{39} \mathrm{~K}\right), \mathbf{I}_{A}$ - величина ядерного спина частицы $A$ (атомы ${ }^{23} \mathrm{Na}$ ) равна $3 / 2$, $\overline{\bar{\gamma}}_{A B}$ и $\overline{\bar{\gamma}}_{A A}-$ мнимые части комплексной скорости спинового обмена $\gamma$, которая может быть выражена через мнимые части комплексного сечения спинового обмена $\left(\gamma_{A B}=\left\langle v_{A B}\right\rangle N_{B} \sigma_{A B}, N_{B}-\right.$ концентрация частиц сорта $B$ (в нашем случае - атомов $\left.{ }^{39} \mathrm{~K}\right),\left\langle v_{A B}\right\rangle-$ средняя относительная тепловая скорость сталкивающихся частиц, $\sigma_{A B}-$ сечение спинового обмена сталкивающихся частиц), $\bar{\omega}_{0}=H_{0}\left|g_{S}\right| \mu_{B} \hbar^{-1}$ - частота электронной прецессии, $H_{0}$ - постоянное магнитное поле, $g_{s}-g$ фактор электрона, $\mu_{B}$ - магнетон Бора. Индексы $A A$ и $A B$ соответствуют столкновениям между атомами $\mathrm{Na}$ или атомами $\mathrm{Na}$ и $\mathrm{K}$, величины $B_{ \pm}$в соответствии с [10] имеют вид

$$
\begin{gathered}
B_{+}=\frac{2 I_{A}+2}{6}\left(\frac{2 I_{A}+3}{2 I_{A}+1}\right)^{1 / 2}, \\
B_{-}=\frac{2 I_{A}}{6}\left(\frac{2 I_{A}-1}{2 I_{A}+1}\right)^{1 / 2}, \\
C=\frac{2 I_{A}\left(2 I_{A}+2\right)\left(2 I_{A}+3\right)\left(2 I_{A}-1\right)}{288\left(2 I_{A}+1\right)^{4}} .
\end{gathered}
$$

Таким образом, для расчета интересующих нас сдвигов частоты магнитного резонанса, обусловленных спиновым обменом в соответствии с (4)-(6) нам необходимо знать величины комплексных сечений спинового обмена (в первую очередь их мнимых частей, а в случае сдвига, определяемого соотношением (6), еще и действительных частей комплексных сечений) для следующих пар атомов: $\mathrm{Na}-\mathrm{Na}, \mathrm{Na}-\mathrm{K}$.

\section{2. Комплексные сечения спинового обмена при столкновении атомов $\mathrm{Na}$ и $\mathrm{K}$}

При столкновении двух щелочных атомов образуется димер, который может быть описан соответствующими потенциалами взаимодействия. Поскольку основному состоянию щелочных атомов соответствует электронный спин $\mathbf{S}=1 / 2$, образующийся в процессе столкновения димер может быть описан с помощью двух потенциалов, соответствующих полным электронным спинам $\mathbf{S}=0$ (синглетный терм $X^{1} \Sigma^{+}$) и $\mathbf{S}=1$ (триплетный терм $a^{3} \Sigma^{+}$). Учет только электронного состояния при расчете потенциалов взаимодействия обусловлен тем, что время столкновения атомов $\sim 10^{-12} \mathrm{~s}$, в то время как время сверхтонкого взаимодействия $2 \pi / \Delta \omega$ для щелочных атомов существенно больше (например, для атомов ${ }^{23} \mathrm{Na}$ $\left.\Delta v=1717 \cdot 10^{6} \mathrm{~Hz}[17]\right)$. Таким образом, за время столкновения не возникает связи между электронными и ядерными степенями свободы системы, а только между электронными. Распределение поляризации между электронными и ядерными степенями свободы атома происходит в интервале между столкновениями.

Для того, чтобы рассчитать интересующие нас сдвиги частоты магнитного резонанса (4)-(6), необходимо знать комплексные сечения спинового обмена соответственно для димера $\mathrm{Na}_{2}$ и димера $\mathrm{NaK}$. Сечения были ранее рассчитаны в [12] для димера $\mathrm{Na}_{2}$ и в [13] для димера $\mathrm{NaK}$. Комплексные сечения спинового обмена имеют вид [18]:

$$
q=\bar{q}+i \overline{\bar{q}}
$$

Данное сечение может быть представлено стандартным образом через фазы рассеяния $\left(\delta_{0,1}(l)\right)$ соответственно на синглетном или триплетном термах для пар $\mathrm{Na}-\mathrm{K}$ и $\mathrm{Na}-\mathrm{Na}$.

Принимая во внимание результаты [18], действительную и мнимую части комплексного сечения можно представить через фазы рассеяния в виде:

$$
\begin{aligned}
& \bar{q}=\frac{\pi}{k^{2}} \sum_{l=0}^{\infty}(2 l+1) \sin ^{2}\left[\delta_{1}(l)-\delta_{0}(l)\right], \\
& \overline{\bar{q}}=\frac{\pi}{k^{2}} \sum_{l=0}^{\infty}(2 l+1) \sin ^{2}\left[\delta_{1}(l)-\delta_{0}(l)\right] .
\end{aligned}
$$

Здесь $k=\mu \nu / \hbar-$ волновой вектор, $l$ - орбитальное квантовое число.

Таким образом, для расчета интересующих нас сечений необходимо рассчитать фазы рассеяния на соответствующих термах димеров $\mathrm{Na}_{2}, \mathrm{NaK}$ и затем, используя 


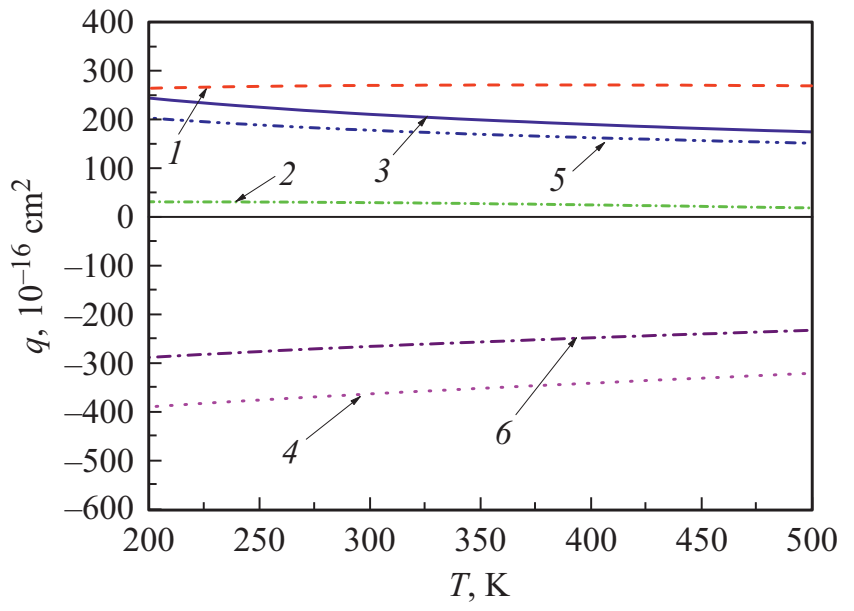

Рис. 1. Температурные зависимости действительной и мнимой частей комплексного сечения спинового обмена: 1 - действительная часть комплексного сечения спинового обмена при столкновении атомов Na и К по данным [13] для потенциалов взаимодействия из $[16] ; 2-$ мнимая часть комплексного сечения спинового обмена при столкновении атомов $\mathrm{Na}$ и $\mathrm{K}$ по данным [13] для потенциалов взаимодействия из [16]; 3 - действительная часть комплексного сечения спинового обмена при столкновении атомов $\mathrm{Na}$ по данным [12] для потенциалов взаимодействия из [15] - синглетный потенциал, из [14] - триплетный потенциал; 4 - мнимая часть комплексного сечения спинового обмена при столкновении атомов $\mathrm{Na}$ и $\mathrm{Na}$ по данным [12] для потенциалов взаимодействия из [15] - синглетный потенциал, из [14] - триплетный потенциал; 5 - действительная часть комплексного сечения спинового обмена при столкновении атомов Na по данным [12] для потенциалов взаимодействия из [15]; 6 - мнимая часть комплексного сечения спинового обмена при столкновении атомов $\mathrm{Na}$ и $\mathrm{Na}$ по данным [12] для потенциалов взаимодействия из [15].

полученные величины, рассчитать интересующие нас комплексные сечения спинового обмена.

Расчет комплексных сечений спинового обмена при столкновении двух атомов $\mathrm{Na}$ проводился в [12] с использованием потенциалов взаимодействия из $[14,15]$ и [13] для системы $\mathrm{Na}-\mathrm{K}$ с учетом потенциалов взаимодействия из работы [16]. На рис. 1 приведены температурные зависимости действительных и мнимых частей комплексного сечения спинового обмена (9). Приведенные на рис. 1 зависимости получены в результате максвелловского усреднения рассчитанных в $[12,13]$ энергетических зависимостей комплексных сечений спинового обмена.

\section{3. Сдвиги частоты магнитного резонанса атомов натрия при столкновении с атомами калия}

Для расчета сдвигов частоты магнитного резонанса в соответствии с формулами (4)-(6) необходимо опре- делить константы скорости спинового обмена, которые зависят от скорости сталкивающихся частиц, величин соответствующих сечений, а также от концентрации сталкивающихся атомов $\left(\gamma_{A B}=\left\langle v_{A B}\right\rangle N_{B} \sigma_{A B}\right)$. Концентрация щелочных атомов определялась в соответствии с данными, приведенными в [19]. Поскольку в эксперименте использовалась смесь щелочных атомов, для перехода от температуры в камере поглощения к концентрации в ней щелочных атомов необходимо воспользоваться законом Рауля для давления насыщенного пара над расплавом смеси металлов. При расчете предполагалось, что щелочные атомы калия и натрия присутствуют в камере поглощения в равных весовых частях.

В дальнейшем расчет температурных зависимостей величин сдвигов $\delta^{(1)} \omega(+)$ и $\delta^{(1)} \omega(-)$ проводился в соответствии с выражениями (4), (5) и (7). На рис. 2 приведены температурные зависимости величин сдвигов при различных значениях степени поляризации атомов $\mathrm{K}$ (величина $P_{z}$ ), рассчитанные с использованием потенциалов взаимодействия из $[14,15]$ для димера $\mathrm{Na}_{2}$ и из [16] для димера $\mathrm{NaK}$. Как правило, в экспериментах по оптической ориентации щелочных атомов поляризация атомов достигает величины нескольких десятков процентов. Представленные на рис. 2 зависимости построены при величинах $P_{z}=100,50$ и 20\%. Как следует из рисунка, в исследуемом интервале температур величины сдвигов $\delta^{(1)} \omega(-)$ возрастают по величине и растут в

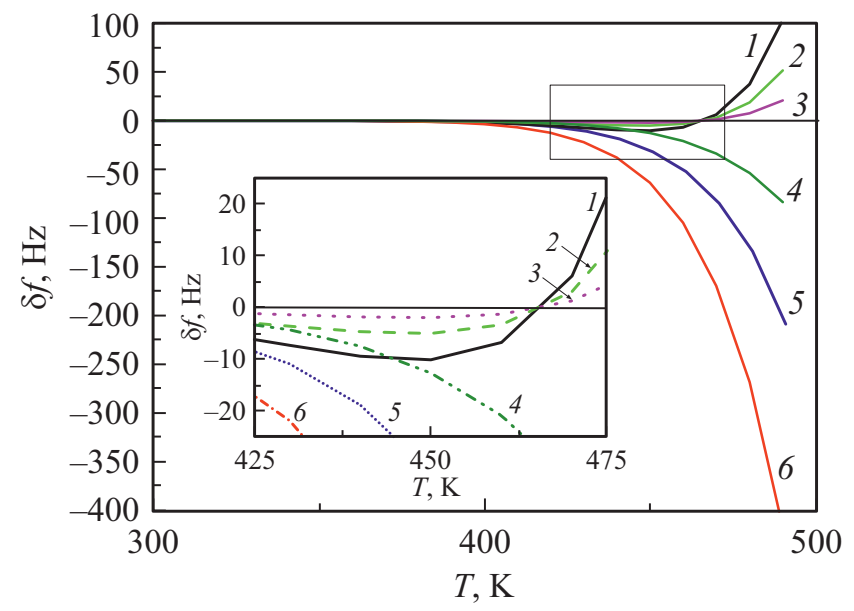

Рис. 2. Температурные зависимости сдвигов частоты магнитного резонанса атомов натрия в основном состоянии, обусловленные спиновым обменом в смеси $\mathrm{Na}-\mathrm{K}$ с использованием потенциалов из $[14,15]$ для пары $\mathrm{Na}-\mathrm{Na}$ и из [16] для пары $\mathrm{Na}-\mathrm{K} .1-$ сдвиг частоты $\delta f=\delta \omega^{(1)}(-) / 2 \pi$ при $P_{z}=100 \%$; 2 - сдвиг частоты $\delta f=\delta \omega^{(1)}(-) / 2 \pi$ при $P_{z}=50 \% ; 3-$ сдвиг частоты $\delta f=\delta \omega^{(1)}(-) / 2 \pi$ при $P_{z}=20 \% ; 4-$ сдвиг частоты $\delta f=\delta \omega^{(1)}(+) / 2 \pi$ при $P_{z}=20 \% ; 5-$ сдвиг частоты $\delta f=\delta \omega^{(1)}(+) / 2 \pi$ при $P_{z}=50 \% ; 16-$ сдвиг частоты $\delta f=\delta \omega^{(1)}(+) / 2 \pi$ при $P_{z}=100 \%$. На вставке - температурные зависимости сдвигов частоты магнитного резонанса атомов натрия в основном состоянии, обусловленные спиновым обменом в смеси $\mathrm{Na}-\mathrm{K}$ в интервале температур $350-450 \mathrm{~K}$ в увеличенном масштабе. 
сторону положительных значений, в то же время сдвиги $\delta^{(1)} \omega(+)$ растут по абсолютной величине и остаются отрицательными во всем диапазоне температур. Однако если построить представленные на рис. 2 зависимости в более мелком масштабе, то можно отметить следующее поведение сдвигов $\delta^{(1)} \omega(-)$ и $\delta^{(1)} \omega(+)$ в районе $T=450 \mathrm{~K}$. Величина сдвига $\delta^{(1)} \omega(+)$ отрицательна во всем диапазоне температур, в то время как $\delta^{(1)} \omega(-)$ растет по абсолютной величине, оставаясь отрицательной, до температур в районе $430 \mathrm{~K}$. Затем величина сдвига начинает уменьшаться по абсолютной величине, но остается отрицательной до диапазона температур 440-460 К. В интервале температур 460-470 К сдвиг частоты проходит точку „0“ и начинает резко расти по величине, становясь положительным. На вставке рис. 2 представлены зависимости исследуемых сдвигов в более мелком масштабе, который позволяет пронаблюдать обнаруженную особенность.

На рис. 3 и вставке на этом рисунке приведены аналогичные зависимости для случая, когда для расчетов использовались потенциалы для димера $\mathrm{Na}_{2}$ из [15]. В этом случае переход через „0“ происходит в интервале температур 440-450 K, т. е. точка переполюсовки сдвига частоты $\delta^{(1)} \omega(-)$ сдвигается в сторону более низких температур.

На рис. 4 приведен расчет температурной зависимости сдвига частоты $\delta^{(2)} \omega=2 \pi \delta f^{(2)}$, определяемого соотно-

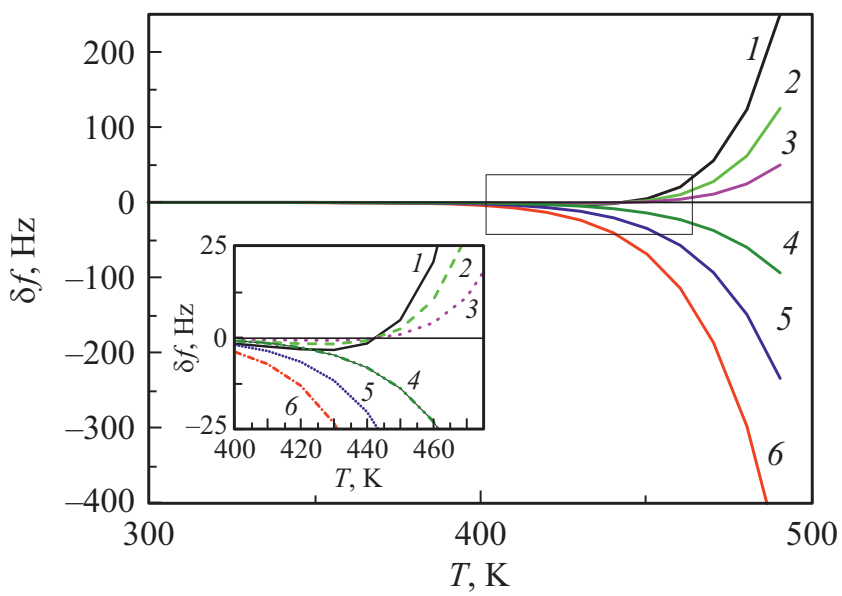

Рис. 3. Температурные зависимости сдвигов частоты магнитного резонанса атомов натрия в основном состоянии, обусловленные спиновым обменом в смеси $\mathrm{Na}-\mathrm{K}$ с использованием потенциалов из [15] для пары $\mathrm{Na}-\mathrm{Na}$ и из [16] для пары $\mathrm{Na}-\mathrm{K} .1-$ сдвиг частоты $\delta f=\delta \omega^{(1)}(-) / 2 \pi$ при $P_{z}=10 \% ; 2-$ сдвиг частоты $\delta f=\delta \omega^{(1)}(-) / 2 \pi$ при $P_{z}=50 \% ; \quad 3-$ сдвиг $\quad$ частоты $\quad \delta f=\delta \omega^{(1)}(-) / 2 \pi \quad$ при $P_{z}=20 \% ; \quad 4-$ сдвиг частоты $\delta f=\delta \omega^{(1)}(+) / 2 \pi \quad$ при $P_{z}=20 \% ; 5-$ сдвиг частоты $\delta f=\delta \omega^{(1)}(+) / 2 \pi \quad$ при $P_{z}=50 \% ; \quad 6-$ сдвиг частоты $\delta f=\delta \omega^{(1)}(+) / 2 \pi \quad$ при $P_{z}=100 \%$. На вставке - температурные зависимости сдвигов частоты магнитного резонанса атомов натрия в основном состоянии, обусловленные спиновым обменом в смеси $\mathrm{Na}-\mathrm{K}$ в интервале температур $350-450 \mathrm{~K}$ в увеличенном масштабе.

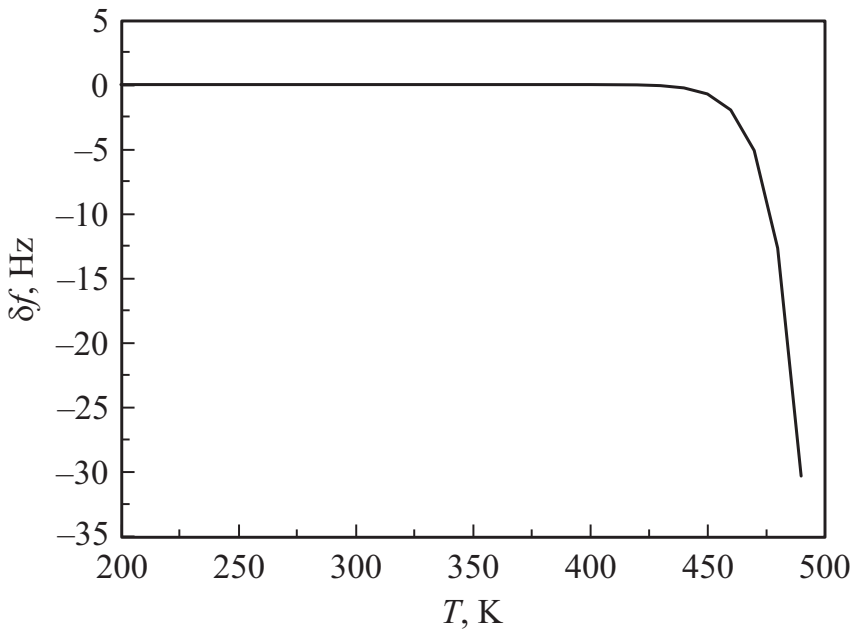

Рис. 4. Температурная зависимость сдвига частоты магнитного резонанса $\left(\delta f=\delta \omega^{(2)} / 2 \pi\right.$ при $\left.P_{z}=100 \%\right)$ атомов натрия в основном состоянии, обусловленного спиновым обменом, в смеси $\mathrm{Na}-\mathrm{K}$ с использованием потенциалов из [15] для пары $\mathrm{Na}-\mathrm{Na}$ и из [16] для пары $\mathrm{Na}-\mathrm{K}$.

шениями (6) и (8) для температуры камеры поглощения в интервале $T=200-500 \mathrm{~K}$ и степени поляризации атомов $P_{z}=100 \%$. Следует отметить, что величина сдвига очень слабо зависит от степени поляризации атома $B$ (атом K), так как в первую очередь определяется первой круглой скобкой в выражении (6). Величина в круглой скобке более чем на порядок превосходит величину в квадратной скобке. Это связано как с соотношением величин действительных и мнимых частей сечений (рис. 1), так и с соотношением концентраций атомов $\mathrm{Na}$ и К. Кроме того, для исследуемой системы атомов основную роль играет второе слагаемое в круглых скобках. Во-первых, как видно из рис. 1, сечение $\sigma_{A B}$ (действительная часть) больше по величине, чем сечение $\sigma_{A A}$ (действительная часть). Во-вторых, в камере поглощения концентрация атомов $\mathrm{K}$ выше, чем концентрация атомов $\mathrm{Na}$, более, чем на порядок, при всех температурах. Эти факторы определяют поведение величины $\delta^{(2)} \omega=2 \pi \delta f^{(2)}$ от температуры.

\section{Заключение}

В заключение следует отметить следующее. При расчете сдвигов частоты магнитного резонанса для других пар щелочных атомов, например K-Cs [20], не было обнаружено изменения знака температурной зависимости сдвигов частоты как для величины $\delta^{(1)} \omega(+)$, так и для величины $\delta^{(1)} \omega(-)$ во всем температурном диапазоне. Однако ранее в [10] было обнаружено „зануление“ сдвига частоты магнитного резонанса и прохождение его через „0“ для случая атомов цезия в смеси цезийрубидий. В работе учитывалось наличие буферного газа в камере поглощения. В результате „зануление“ величины сдвига $\delta^{(1)} \omega(-)$ наблюдалось при изменении 
давления буферного газа в камере поглощении и для $\delta^{(1)} \omega(+)$ - при изменении температуры камеры поглощения при условии присутствия буферного газа.

Наблюдавшееся в настоящей работе изменение знака сдвига частоты магнитного резонанса $\delta^{(1)} \omega(-)$ обусловлено конкуренцией двух процессов. Как следует из формулы (5), вклад в величину сдвига вносят два спин-обменных процесса, конкурирующих друг с другом: первый процесс - это столкновение атомов натрия с атомами калия (вклад от этого процесса определяется первым слагаемым в квадратной скобке выражения (5)), второй процесс - столкновение атомов натрия между собой (второй член в квадратной скобке выражения (5)). В свою очередь, рассматриваемые слагаемые определяются величинами $\overline{\bar{\gamma}}_{A A}$ и $\overline{\bar{\gamma}}_{A B}$ (где $\overline{\bar{\gamma}}_{A B}=\left\langle v_{A B}\right\rangle N_{B} \overline{\bar{\sigma}}_{A B}$ и $\left.\overline{\bar{\gamma}}_{A A}=\left\langle v_{A A}\right\rangle N_{A} \overline{\bar{\sigma}}_{A A}\right)$. Здесь индекс $A A$ относится к столкновению атомов натрия между собой, а индекс $A B-$ к столкновению атомов натрия и калия. Как следует из данных рис. 1, мнимая часть сечения спинового обмена отрицательна во всем диапазоне рассматриваемых температур для случая столкновения атомов натрия между собой, в то время как мнимая часть сечения спинового обмена для столкновения атомов натрия и калия имеет положительный знак. С ростом температуры в камере поглощения растет концентрация атомов калия и натрия, а также изменяется величина сечений спинового обмена. Таким образом, в области более низких температур величина сдвига частоты магнитного резонанса $\delta^{(1)} \omega(-)$ определяется вторым слагаемым в формуле (5) (зависит от спинового обмена между атомами натрия). Это слагаемое имеет отрицательное значение. По мере роста температуры все большую роль начинает играть первое слагаемое в (5) (определяется спиновым обменом между атомами натрия и калия), которое имеет положительный знак. Конкуренция между этими двумя слагаемыми, из которых первое растет быстрее, и приводит к тому, что в температурной зависимости наблюдается смена знака с отрицательного на положительный и температурная зависимость величины сдвига частоты магнитного резонанса $\delta^{(1)} \omega(-)$ проходит через ноль.

В то же время в формуле (4) величина в квадратной скобке имеет положительное значение во всем исследуемом интервале температур, так как в соответствии с рис. 1 мнимые части комплексных сечений (9) для пар $\mathrm{Na}-\mathrm{Na}$ и $\mathrm{Na}-\mathrm{K}$ имеют разные знаки, отрицательный для двух натриев и положительный для натрия и калия, причем вклад от спинового обмена между двумя атомами натрия входит со знаком (-). Таким образом, квадратная скобка в выражении (4) имеет положительный знак, а учет знака (-) перед ней в выражении (4) приводит к тому, что сдвиг частоты магнитного резонанса $\delta^{(1)} \omega(+)$ имеет отрицательный знак и растет по величине с ростом температуры.

Следует отметить, что все приборы квантовой электроники, в основу работы которых положен принцип оптической ориентации атомов, как то квантовые магнитометры, стандарты частоты, квантовые гироскопы, квантовые магнитоэнцифалорафы (на основе квантовых магнитометров) сталкиваются с проблемой, связанной со сдвигами частоты магнитного резонанса. Это обусловлено тем, что в этих устройствах происходит измерение частоты магнитного резонанса. Истинное значение измеряемой величины может быть искажено величиной сдвига резонансной частоты, обусловленного различными физическими процессами: спиновым обменом, влиянием света накачки, столкновения с атомами буферного газа и т.д. Таким образом, зная возможное поведение сдвига частоты магнитного резонанса, в данном случае зависящего от спинового обмена при столкновении поляризованных щелочных атомов, можно подобрать условия, а также соответствующие смеси щелочных атомов для минимизации отрицательного влияния сдвига частоты на точностные, характеристики устройств.

\section{Конфликт интересов}

Авторы заявляют, что у них нет конфликта интересов.

\section{Список литературы}

[1] D. Budker, M. Romalis. Nature Phys., 3 (4), 227 (2007).

[2] Е.Б. Александров, А.К. Вершовский. УФН, $179(6), 605$ (2009). [E.B. Aleksandrov, A.K. Vershovskii. Phys. Usp., 52, 605 (2009).]

[3] T.G. Walker, M.S. Larsen. Advances In Atomic, Molecular, and Optical Physics, 65, 373 (2016). https://doi.org/10.1016/bs.aamop.2016.04.002

[4] E. Boto, S.S. Meyer, V. Shah, O. Alem, S. Knappe, P. Kruger, T.M. Fromhold, M. Lim, P.M. Glover, P.G. Morris, R. Bowtell, G.R. Barnes, M.J. Brookes. NeuroImage, 149, 404 (2017). DOI: http://dx.doi.org/10.1016/j.neuroimage.2017.01.034

[5] S. Groeger, G. Bison, J.-L. Schenker, R. Winands, A. Weis. Eur. Phys. J. D, 38 (2), 239 (2006). DOI: 10.1140/epjd/e2006-00037-y

[6] Y. Chen, W. Quan, L. Duan, Y. Lu, L. Jiang, J. Fang. Phys. Rew. A, 94, 052705 (2016) DOI: 10.1103/PhysRevA.94.052705

[7] Y. Lu, Y. Zhang, W. Fan, L. Xing, Y. Zhai, W. Quan. Chinese Phys. B, 29 (4), 043204 (2020). DOI: $10.1088 / 1674-1056 / \mathrm{ab} 75 \mathrm{~d} 3$

[8] С.П. Дмитриев, Н.А. Доватор, В.А. Картошкин. ЖТФ, 85 (6), 40 (2015). [S.P. Dmitriev, N.A. Dovator, V.A. Kartoshkin. Tech. Phys., 60 (6), 826 (2015). DOI: http://dx.doi.org/10.1134/S1063784215060079]

[9] K. Wei, T. Zhao, X. Fang, H. Li, Y. Zhai, B. Han, W. Quan. Phys. Rev. Appl., 13 (4), 044027 (2020). DOI: 10.1103/PhysRevApplied.13.044027

[10] А.И. Окуневич. Опт. и спектр., 79(5), 718 (1995). [A.I. Okunevich. Opt. Spectr., 79 (5), 718 (1995).]

[11] P. Bicchi, L. Mon, P. Savino, B. Zambon. Il Nuovo Chimento, 55|,(1), 1 (1980). DOI: https://doi.org/10.1007/BF02728372 
[12] В.А. Картошкин. Опт. и спектр., 116 (4), 588 (2014). [V.A. Kartoshkin. Opt. Spectr., 116 (4), 548 (2014). DOI: http://dx.doi.org/10.1134/S0030400X14030096]

[13] B.A. Картошкин. Опт. и спектр., 109(5), 733 (2010). [V.A. Kartoshkin. Opt. Spectr., 109 (5), 674 (2010). DOI: http://dx.doi.org/10.1134/S0030400X10110056]

[14] V.S. Ivanov, V.B. Sovkov. J. Chem. Phys., 118(18), 8242 (2003). DOI: https://doi.org/10.1063/1.1565107

[15] W.T. Zemke, W.C. Stwalley. J. Chem. Phys., 100 (4), 2661 (1994). DOI: https://doi.org/10.1063/1.467249

[16] A. Gerdes, M. Hobein, H. Knöckel, E. Tiermann. Eur. Phys. J. D, 49 (1), 67 (2008). DOI: 10.1140/epjd/e2008-00138-7

[17] Н.М. Померанцев, В.Н. Рыжков, Г.В. Скроцкий. Физические основы квантовой магнитометрии (Наука, M.,1972)

DOI: http://dx.doi.org/10.1134/S1063784215060079

[18] Н. Мотт, Г. Месси. Теория атомных столкновений (Мир, M., 1969)

[19] А.Н. Несмеянов. Давление паров химических элементов (Изд-во АН, М., 1961).

[20] В.А. Картошкин. Опт. и спектр., 128 (9), 1244 (2020).

DOI: http://dx.doi.org/10.21883/OS.2020.09.49859.125-20

[V.A. Kartoshkin. Opt. Spectr., $128(9), 1355$ (2020).

DOI: http://dx.doi.org/10.1134/S0030400X2009012X] 